\title{
Parasites Gastro-Intestinaux des Populations Humaines du Parc National de Taï, Côte d'Ivoire
}

\author{
Kouassi Wa Yao Roland, PhD \\ Laboratoire de Zoologie et Biologie Animale, UFR Biosciences, \\ Université Félix Houphouët-Boigny, Abidjan, Côte d'Ivoire \\ Perrotey Sylvie, PhD \\ Laboratoire de Parasitologie, Faculté de Pharmacie, \\ Université de Strasbourg, France
}

Bassa Kouakou Fidèle, PhD

Laboratoire de Zoologie et Biologie Animale, UFR Biosciences,

Université Félix Houphouët-Boigny, Abidjan, Côte d'Ivoire

\section{Bohoussou Kouakou Hilaire, PhD}

UFR Ingénierie Agronomique Forestière et Environnementale,

Université de Man, Côte d'Ivoire

N'goran Kouakou Eliezer, PhD

Laboratoire de Zoologie et Biologie Animale, UFR Biosciences, Université Félix Houphouët-Boigny, Abidjan, Côte d'Ivoire

Doi:10.19044/esj.2019.v15n36p27 URL:http://dx.doi.org/10.19044/esj.2019.v15n36p27

\section{Résumé}

Les affections parasitaires sévissent dans les pays en voie de développement, en particulier au sein des populations pauvres des régions tropicales. En Côte d'Ivoire, des précédents travaux ont montré des taux de prévalence élevés pour les infestations parasitaires intestinales. Le but de notre étude était d'investiguer sur la présence des parasites gastro-intestinaux chez les populations de 3 villages situés autour du parc national de Taï (PNT) ainsi que chez les chercheurs et les assistants de recherche travaillant au sein du PNT. L'analyse de 586 échantillons de selles par la méthode de Ritchie et par la technique de concentration Merthiolate-Iodine-Formaldehyde (MIF) a mis en évidence la présence de 21 parasites dont 12 helminthes et 9 protozoaires. Les prévalences parasitaires globales étaient élévées $(69,1 \%)$ dans l'ensemble des sites échantillonnés. Les prévalences parasitaires globales étaient élévées $(69,1 \%)$ dans l'ensemble des sites échantillonnés. Le village de Gouléako 1 a enregistré la prévalence parasitaire globale la plus élevée (81,43\%), et le vilage de Paulé Oula la plus faible (64,58\%). Les individus de la classe d'âge [7-14ans] étaient les plus infestés avec un pic d'infestations pendant la saison pluvieuse. Le polyparasitisme est très marqué avec $89 \%$ des 
sujets infestés par au moins deux espèces de parasites. L’inventaire faunistique des parasites intestinaux des populations humaines vivant à proximité du PNT a permis de rajouter six nouvelles espèces à la liste des parasites identifiés chez les populations de Côte d'Ivoire. Il s'agit d'Oesophagostomum sp., de Capillariidae Gen. sp. 2, de Dicrocoelium sp., de Trichostrongylus sp., de Strongyloides fuelleborni et de Balantidium coli.

Mots-clés: Parasites, Prévalence, Communauté rurale, Côte d'Ivoire

\title{
Gastrointestinal Parasites from Humans Populations of Taï National Park, Côte d'Ivoire
}

\author{
Kouassi Wa Yao Roland, PhD \\ Laboratoire de Zoologie et Biologie Animale, UFR Biosciences, \\ Université Félix Houphouët-Boigny, Abidjan, Côte d'Ivoire \\ Perrotey Sylvie, PhD \\ Laboratoire de Parasitologie, Faculté de Pharmacie, \\ Université de Strasbourg, France
}

Bassa Kouakou Fidèle, PhD

Laboratoire de Zoologie et Biologie Animale, UFR Biosciences,

Université Félix Houphouët-Boigny, Abidjan, Côte d'Ivoire

\section{Bohoussou Kouakou Hilaire, PhD}

UFR Ingénierie Agronomique Forestière et Environnementale,

Université de Man, Côte d'Ivoire

N'goran Kouakou Eliezer, PhD

Laboratoire de Zoologie et Biologie Animale, UFR Biosciences, Université Félix Houphouët-Boigny, Abidjan, Côte d'Ivoire

\begin{abstract}
Parasitic diseases are prevalent in developing countries, especially among poor communities in the tropics. In Côte d'Ivoire, previous studies have recorded high prevalence for intestinal parasite infestations. This paper focuses on investigating the presence of gastro-intestinal parasites in rural populations from 3 villages located near the Tai National Park (TNP) and among researchers and research assistants working in TNP. Screening of 586 stool samples by Ritchie method and Merthiolate-Iodine-Formaldehyde concentration technique has revealed the presence of 21 parasites including 12 helminths and 9 protozoa. The overall parasite prevalence was high $(69.1 \%)$.
\end{abstract}


We found the highest and lowest prevalence in Gouléako 1 (81.43\%) and Paule Oula (64.58\%) villages respectively. The age group [7-14] has been more infested with a peak of infestations during rainy season. Polyparasitism is very marked with $89 \%$ of individuals harboring at least two species of parasites. The intestinal parasites inventory, detected in rural populations living near the PNT, allowed us to add six species to the list of parasites identified in the human population of Côte d'Ivoire. These are Oesophagostomum sp., Capillariidae Gen. sp., 2 Dicrocoelium sp., Trichostrongylus sp., Strongyloides fuelleborni and Balantidium coli.

Keywords: Parasites, Prevalence, Rural community, Côte d'Ivoire

\section{Introduction}

Les maladies infectieuses dues à des parasites gastro-intestinaux représentent un important problème de santé publique. Environ 2 milliards de personnes sont touchées dans le monde. C'est notamment le cas des populations rurales pauvres de 1'Afrique subsaharienne, de 1'Asie orientale et

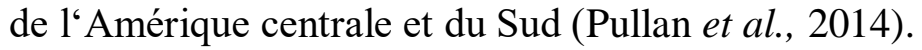

L'intensité saisonnière et la distribution de ces infections parasitaires sont fortement influencées par les facteurs géographiques, les conditions climatiques et hygiéniques trop souvent précaires et les activités humaines qui entraine la modification de l'environnement et l'apparition de pathogènes émergentes (Wilcox \& Gubler, 2005).

En Côte d'Ivoire, le Parc National de Taï (PNT) représente la plus vaste aire pleinement protégée du massif forestier ouest-africain et constitue un grand centre de diversité pour de nombreuses espèces animales et végétales (Schweter, 1999). Cependant, la partie ouest du PNT est bordée par plusieurs villages souvent très proches du PNT, du fait de leur confinement entre le fleuve Cavally à l'ouest et le PNT à l'est. Cette région est aussi caractérisée par une grande diversité de populations humaines dont l'activité principale est l'agriculture, avec un mélange de cultures vivrières et de cultures de rentes (Leonard \& Ibo, 1994).

Depuis quelques années, des maladies bactériennes et virales ont été signalées chez les primates du PNT et dans les populations riveraines (Leendertz et al., 2004 ; Köndgen et al., 2008 ; Mossoun et al., 2015; Anoh et al., 2015; 2017). Un nombre limité de travaux s'est interessé aux parasites gastro-intestinaux que les chez primates non-humains du PNT (Kouassi et al., 2015). A ce jour, à notre connaissance, aucune étude ne s'est focalisée sur ceux des populations humaines.

L'objectif de ce travail est donc de dresser un inventaire systématique des parasites gastro-intestinaux des populations rurales du PNT et de définir les facteurs de variation de ces parasites. Il s'agit de la première étude 
consacrée à l'ensemble des parasites gastro-intestinaux des populations humaines de cette région vivant à proximité de l'aire protégée de Taï.

\section{Approche Methodologique}

\section{Site d'étude}

L'étude a été conduite dans la partie ouest du Parc National de Taï et dans 3 villages environnants (Paulé-Oula, Gouléako 1 et Gouléako 2) (Figure 1). Ce site d'étude est localisé dans le sud-ouest de la Côte d'Ivoire (Afrique de l'Ouest) entre les latitudes $5^{\circ} 10$ et $6^{\circ} 10$ nord et les longitudes $4^{\circ} 20$ et $6^{\circ} 50$ ouest (Hoppe-Dominik, 1995). Il se trouve à $20 \mathrm{~km}$ à l'est de la ville de Taï. Le PNT est soumis à un climat de type subéquatorial de transition. Le régime pluviométrique est de type bimodal et compte deux grandes saisons pluvieuses. L'une s'étend de septembre à novembre, l'autre d'avril à juin. Elles sont séparées par une inflexion entre juillet et août. La saison sèche débute en décembre et prend fin en mars. La moyenne des précipitations annuelles est de $1800 \mathrm{~mm}$ et la température moyenne mensuelle varie très faiblement entre $24^{\circ} \mathrm{C}$ et $26^{\circ} \mathrm{C}$. L'hygrométrie reste élevée toute l'année ( 85 à 90\%). Elle atteint souvent 100\% (Hoppe-Dominik, 1995).

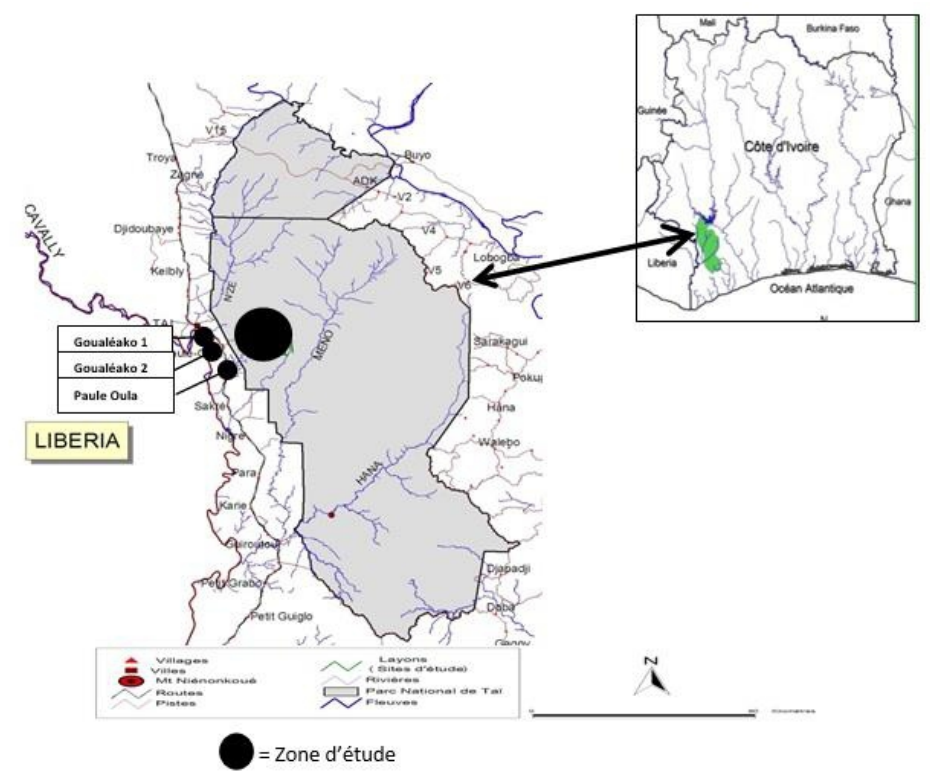

Figure 1. Localisation de la zone d'étude, en Côte d'Ivoire

\section{Populations d'étude}

Des personnes volontaires issues de 3 villages (Paulé-Oula, Gouléako 1, Gouléako 2) situés dans la partie ouest du parc, et des acteurs permanents du PNT (chercheurs et assistants de terrain) ont fait l'objet d'un échantillonnage. 
Tous les participants ont été informés des objectifs et des approches méthodologiques au moyen d'une lettre d'information et de séances d'explications collectives ou individuelles. Après avoir pris connaissance du contenu du projet, chaque individu a décidé librement d'y participer ou non. La participation des enfants mineurs a été soumise à l'autorisation des parents ou tuteurs légaux. L'accord de participation a été matérialisé par l'émargement d'une fiche de consentement éclairé.

\section{Approbation éthique}

Cette étude a reçu l'autorisation de la Direction de la Recherche du Ministère de l'Enseignement Supérieur et de la Recherche Scientifique, de l'Office Ivoirien des Parcs et Réserves (OIPR) du Ministère de l'Environnement et des Eaux et Forêts, et l'approbation du Comité National d'Ethique et de la Recherche (CNER) du Ministère de la Santé (Nº9910/CNER/MSHP). Un code a été attribué à chaque échantillon pour garantir la confidentialité des données. Le traitement des parasitoses intestinales des participants de tous les sites échantillonnés a été effectuaé par l'Albendazole $400 \mathrm{mg}$, à raison d'un comprimé par personne pour tous les cas dépistés, à l'exception des femmes enceintes, conformément au protocole approuvé par le CNER.

\section{Collecte des selles et analyses parasitologiques}

Les selles ont été collectées entre janvier et novembre 2010, pendant la saison sèche et la saison pluvieuse. Des pots de prélèvement de $125 \mathrm{~mL}$ ont été remis à chaque participant. Les selles fraîches du jour-même de la collecte des échantillons ont été alors rapportées par les participants. Le Tableau I présente le nombre d'individus échantillonnés selon la localité, le sexe et la classe d'âge. Les échantillons ont été conservés à la fois dans une solution de formol $10 \%$ et dans une solution de SAF (Sodium acetate-Acetic acidFormalin). Les selles ont été mises en suspension dans 3 fois leur volume d'une solution de formol à $10 \%$. Les tubes sont étiquetés, fermés et secoués vigoureusement de façon à obtenir une suspension homogène. Sur l'étiquette sont codifiés, la date, l'heure, la localité, le sexe, l'âge de l'individu échantillonné et le mode de conservation.

Pour la recherche d'helminthes et de protozoaires, chaque échantillon a été soumis à un examen direct à la technique de Ritchie modifiée, et à celle du MIF (Blagg et al., 1955 ; Young et al., 1979; Erdman, 1981). La lecture des lames a été réalisée à l'aide d'un microscope (Leica DM2000 LED), équipé d'un appareil photo (Leica DFC450). L'identification des parasites était basée sur la morphologie des œufs, des kystes et des larves (taille, couleur, forme). 


\section{Analyses statistiques}

Les prévalences parasitaires ont été estimées par facteur de risque: localité, sexe, classe d'âge et saison. Le logiciel STATA software version 10 (Stata Corporation, College station, USA) a servi à cet effet. Les différentes proportions ont été comparées à l'aide du test de Fischer. Les résultats sont considérés comme significatifs au niveau d'incertitude de $5 \%(\mathrm{p}<0,05)$.

L'indice de Shannon (H') et l'indice d'équitabilité (E) ont permis d'évaluer la richesse spécifique et l'abondance des différentes espèces de parasites ainsi que leur répartition équitable au sein des populations humaines (Colwell et al., 2013).

Tableau I. Nombre d'individus échantillonnés par sites

\begin{tabular}{llccccc}
\hline \multicolumn{7}{c}{ Sites } \\
\hline \multirow{2}{*}{ Genre } & Gouléako 1 & Gouléako 2 & Paulé-Oula & PNT & Total \\
& Femme & 40 & 126 & 143 & 10 & 319 \\
& Homme & 30 & 86 & 97 & 54 & 267 \\
& Total & 70 & 212 & 240 & 64 & 586 \\
\hline \multirow{4}{*}{ Classe } & {$[\mathbf{3 - 6}]$} & 19 & 51 & 85 & - & 155 \\
d'âge & {$[\mathbf{7 - 1 4}]$} & 14 & 68 & 62 & - & 144 \\
& {$[\mathbf{1 5 - 2 4}]$} & 21 & 60 & 59 & - & 140 \\
& {$[\mathbf{2 5 ;}+]$} & 16 & 33 & 34 & 64 & 147 \\
& Total & 70 & 212 & 240 & 64 & 586 \\
\hline
\end{tabular}

\section{Resultats}

\section{Inventaire faunistique des parasites}

Dans les 4 sites sélectionnés (Gouléako 1, Gouléako 2, Paulé-Oula et PNT), 821 personnes ont participé à l'enquête-questionnaire à la suite de laquelle 586 échantillons de selles ont été collectés.

\section{Prévalence et diversité parasitaires}

Les prévalences des parasites observés sur chacun des sites et les prévalences cumulées figurent dans le Tableau II. Pour l'ensemble des 4 localités, la prévalence parasitaire globale est de 69,1\%. Gouléako 1 enregistre la prévalence cumulée la plus élevée $(81,43 \%)$ et Paulé-Oula, la plus faible $(64,58 \%)$.

Les prévalences cumulées des protozoaires sont plus élevées que celles des helminthes dans les 4 sites. Celles-ci sont comprises entre 78,57\% et $60 \%$ pour les protozoaires; elles varient entre $64,28 \%$ et $50,42 \%$ pour les helminthes. Dans ce dernier groupe, les géohelminthes, Ancylostoma sp. (38,57\%), Ascaris lumbricoides (18,4\%), Trichuris trichiura (18,57\%) et Oesophagostomum sp. (10,94\%) ont enregistrés des prévalences supérieures à $10 \%$. Les différences de prévalences entre les 4 sites sont très significatives pour Ancylostoma sp. ( $\mathrm{p}=0,002)$, Ascaris lumbricoides $(\mathrm{p}=0,000)$ et 
Hymenolepis nana $(\mathrm{p}=0,000)$. Dans le groupe des protozoaires, les amibes enregistrent des prévalences supérieures ou égales à $20 \%$. Entamoeba histolytica/dispar a une prévalence de 25,26\%. Balantidium coli et Giardia duodenalis, les deux protozoaires ciliés pathogènes, présentent les plus faibles prévalences, 7,34\% et 9,04\%, respectivement. Les prévalences d'Entamoeba coli $(\mathrm{p}=0,025)$ et d'Endolimax nana $(\mathrm{p}=0,000)$ sont significativement différentes dans les 4 sites.

La richesse spécifique la plus élevée est enregistrée dans les villages de Paulé-Oula et Gouléako 1 Le PNT présente la plus faible richesse spécifique. Cependant, l'indice de Shannon est plus élevé dans le PNT (H' = $2,414)$ avec une distribution plus équitable $(\mathrm{E}=0,84)$ des parasites. PauléOula enregistre les indices de Shannon et d'équitabilité les plus faibles $\left(\mathrm{H}^{\prime}=\right.$ 1,78 et $\mathrm{E}=0,59$ ) (Tableau III).

Tableau II. Prévalence des espèces de parasites dans les différents sites d'étude

\begin{tabular}{|c|c|c|c|c|c|c|}
\hline & & $\begin{array}{c}\text { Gouléako1 } \\
\mathrm{n}=70\end{array}$ & $\begin{array}{c}\text { Gouléako2 } \\
\mathrm{n}=212\end{array}$ & $\begin{array}{l}\text { Parc } \\
\mathrm{n}=64\end{array}$ & $\begin{array}{c}\text { Paulé-Oula } \\
\mathrm{n}=240\end{array}$ & p-value \\
\hline & & & Préval & $(\%)$ & & \\
\hline & Ancylostoma sp. & 38,57 & 19,81 & 14,06 & 27,92 & $0,002 *$ \\
\hline & Ascaris lumbricoides & 11,43 & 18,40 & 0 & 9,58 & $0,000 *$ \\
\hline & Capillariidae Gen. sp. 2 & 5,71 & 3,77 & 4,69 & 3,75 & 0,825 \\
\hline & Oesophagostomum sp. & 7,14 & 10,38 & 10,94 & 10,00 & 0,89 \\
\hline 弐 & Strongyloides fuelleborni & 2,86 & 3,77 & 6,25 & 5,42 & 0,688 \\
\hline 3 & Strongyloides stercoralis & 8,57 & 4,72 & 1,56 & 6,25 & 0,291 \\
\hline Z & Trichuris trichiura & 18,57 & 11,79 & 7,81 & 9,58 & 0,177 \\
\hline $\mathbf{E}$ & Trichostrongylus sp. & 5,71 & 0,94 & 3,13 & 1,67 & 0,077 \\
\hline & Dicrocoelium sp. & 4,29 & 4,72 & 9,38 & 2,92 & 0,174 \\
\hline & Enterobius vermicularis & 0 & 3,30 & 0 & 1,67 & 0,268 \\
\hline & Hymenolepis nana & 4,29 & 12,74 & 0 & 4,58 & $0,000 *$ \\
\hline & Schistosoma mansoni & 5,71 & 3,30 & 6,25 & 2,92 & 0,423 \\
\hline & Total & 64,28 & 56,6 & 51,56 & 50,42 & \\
\hline & Entamoeba coli & 61,43 & 42,45 & 54,69 & 51,67 & $0,025^{*}$ \\
\hline & Entamoeba histolytica/dispar & 30,00 & 24,53 & 17,19 & 26,67 & 0,338 \\
\hline$\pi$ & Entamoeba hartmanni & 27,14 & 19,81 & 23,44 & 16,25 & 0,176 \\
\hline 0 & Endolimax nana & 25,71 & 28,77 & 17,19 & 10,42 & $0,000^{*}$ \\
\hline No & Iodamoeba butschlii & 18,57 & 19,34 & 26,56 & 18,75 & 0,548 \\
\hline 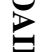 & Balantidium coli & 4,29 & 7,08 & 10,94 & 7,50 & 0,543 \\
\hline 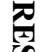 & Chilomastix mesnili & 15,71 & 18,87 & 28,13 & 15,00 & 0,111 \\
\hline & Giardia duodenalis & 11,43 & 7,55 & 12,50 & 8,75 & 0,516 \\
\hline & Blastocystis sp. & 12,86 & 12,26 & 21,88 & 17,08 & 0,214 \\
\hline & Total & 78,57 & 61,79 & 65,63 & 60 & \\
\hline & Totaux & 81,43 & 70,75 & 67,19 & 64,58 & 0,27 \\
\hline
\end{tabular}

* : Différence statitisquement significative 
Tableau III.Richesse spécifique et indices de diversité

\begin{tabular}{lcccc}
\hline Villages & $\begin{array}{c}\text { Richesse } \\
\text { spécifique }\end{array}$ & $\begin{array}{c}\text { Indice de } \\
\text { Shannon } \\
\left(H^{\prime}\right)\end{array}$ & [H'max] & $\begin{array}{c}\text { Indice } \\
\text { d'équitabilité } \\
\text { (E) }\end{array}$ \\
\hline Gouléako 1 & \multicolumn{2}{c}{ n (N) } & Helminthes et Protozoaires \\
Gouléako 2 & $20(21)$ & 1,89 & {$[2,99]$} & 0,6309 \\
PNT & $18(21)$ & 1,973 & {$[3,04]$} & 0,648 \\
Paulé-Oula & $21(21)$ & 2,414 & {$[2,89]$} & 0,8354 \\
\hline
\end{tabular}

$\mathrm{n}$ : Nombre d'espèce de parasites observés dans chaque site

$\mathrm{N}$ : Nombre total d'espèces de parasites observés

E : Indice d'équitabilité de Piélou

H' : Indice de Shannon

[H' max] : Valeur maximale de l'indice de Shannon H' calculé

\section{Corrélations entre les espèces de parasites, l'âge, le sexe et la saison}

Les analyses coprologiques ont permis d'identifier 21 parasites intestinaux dont 12 helminthes et 9 protozoaires.

Les échantillons de selles humaines ont été collectés sur 2 saisons (Saison Pluvieuse : SP et Saison Sèche : SS) et répartis également par sexe et classes d'âge (Tableau IV). Les prévalences parasitaires de ces différentes catégories sont présentées dans le Tableau V.

Les prévalences parasitaires cumulées des hommes et des femmes sont respectivement de $70 \%$ et $69 \%$. Ancylostoma sp., Ascaris lumbricoides et Trichuris trichiura, sont les géohelminthes prédominants dans les deux groupes. Une liaison significative a été observée entre le sexe et les prévalences d'Oesophagostomum $\mathrm{sp} .(\mathrm{p}=0,022)$, de Schistosoma mansoni $(\mathrm{P}$ $=0,003)$, de Blastocystis $\mathrm{sp} .(\mathrm{p}=0,001)$ et de Balantidium coli $(\mathrm{p}=0,049)$.

La classe d'âge [7-14 ans] a présenté la prévalence parasitaire cumulée la plus élevée $(74,29 \%)$ et la classe d'âge [15-25 ans] la prévalence cumulée la plus faible $(66,67 \%)$. Les prévalences d'Ascaris lumbricoides et d'Oesophagostomum sp. ont montré, respectivement, une liaison hautement significative $(\mathrm{P}=0,000)$ et significative $(\mathrm{P}=0,046)$ avec l'âge.

La saison des pluies a enregistré la prévalence cumulée de parasites la plus élevée (69,86\%). Les prévalences de Capillariidae Gen. Sp.1, Endolimax nana, Balantidium coli et Giardia duodenalis présentent une différence significative selon la saison.

\section{Polyparasitisme}

Sur l'ensemble des échantillons prélevés dans les quatre sites, 18 individus $(3,07 \%)$ ne sont infestés par aucun parasite intestinal. 47 individus $(8,02 \%)$ sont infestés par une seule espèce de parasites et 521 individus $(88,91$ $\%$ ) le sont pour au moins 2 espèces de parasites (Tableau VI). 
Les infestations à 3 et 4 espèces de parasites sont les plus prédominantes (Figure 1). Le plus faible taux d'individus non parasités a été enregistré à Gouléako 2. Le taux le plus élevé de polyparasitisme a été enregistré dans les localités de Gouléako 1 et dans le PNT avec des prévalences souvent au-delà de $25 \%$ (Figure 2).

Tableau IV. Répartition des échantillons de selles humaines selon le sexe, la classe d'âge et la saison

\begin{tabular}{llllllllllll}
\hline & Genre & \multicolumn{1}{c}{ Classe d'âge } & \multicolumn{3}{c}{ Saison } \\
\hline & Femme & Homme & Total & {$[\mathbf{3 - 6}]$} & {$[\mathbf{7 - 1 4}]$} & {$[\mathbf{1 5 - 2 4}]$} & {$[\mathbf{2 5} ;+\mathbf{+}$} & Total & SS & SP & Total \\
\cline { 2 - 12 } Gouléako 1 & 40 & 30 & 70 & 19 & 14 & 21 & 16 & 70 & 38 & 32 & 70 \\
Gouléako 2 & 126 & 86 & 212 & 51 & 68 & 60 & 33 & 212 & 148 & 64 & 212 \\
Paulé-Oula & 143 & 97 & 240 & 85 & 62 & 59 & 34 & 240 & 137 & 103 & 240 \\
PNT & 10 & 54 & 64 & - & - & - & 64 & 64 & 32 & 32 & 64 \\
\hline Total & 319 & 267 & 586 & 155 & 144 & 140 & 147 & 586 & 355 & 231 & 586 \\
\hline
\end{tabular}

SS : Saison Sèche

SP : Saison Pluvieuse 
Tableau V. Prévalences des parasites gastro-intestinaux selon le genre, la saison et la classe d'âge

\begin{tabular}{|c|c|c|c|c|c|c|c|c|c|c|c|c|}
\hline & & \multicolumn{3}{|c|}{ Genre } & \multicolumn{5}{|c|}{ Classe d'âge } & \multicolumn{3}{|c|}{ Saison } \\
\hline \multirow{14}{*}{ 焉 } & & $\begin{array}{l}\text { Femme } \\
\mathrm{n}=319\end{array}$ & $\begin{array}{r}\text { Homme } \\
n=267\end{array}$ & p-value & $\begin{array}{c}{[25 \text { et }+]} \\
\mathrm{n}=147\end{array}$ & $\begin{array}{l}{[\mathbf{1 5 - 2 4}]} \\
\mathrm{n}=140\end{array}$ & $\begin{array}{r}{[7-14]} \\
n=144\end{array}$ & $\begin{array}{r}{[3-6]} \\
n=155\end{array}$ & p-value & $\begin{array}{c}\text { SP } \\
n=231\end{array}$ & $\begin{array}{c}\text { SS } \\
\mathrm{n}=355\end{array}$ & p-value \\
\hline & & \multicolumn{11}{|c|}{ Prévalence (\%) } \\
\hline & Ancylostoma sp. & 23,42 & 26,3 & 0,421 & 24,49 & 25 & 27,14 & 22,52 & 0,865 & 27,71 & 22,82 & 0,18 \\
\hline & Ascaris lumbricoides & 13,29 & 10,37 & 0,277 & 2,04 & 10,14 & 15,71 & 19,87 & $0.000^{*}$ & 12,99 & 11,27 & 0,531 \\
\hline & Capillariidae Gen. sp. 2. & 3,48 & 4,81 & 0,417 & 2,72 & 4,73 & 3,57 & 5,3 & 0,672 & 6,49 & 2,54 & $0,018 *$ \\
\hline & Oesophagostomum sp. & 7,28 & 12,96 & $0,022 *$ & 10,88 & 5,41 & 15 & 8,61 & $0,046^{*}$ & 11,69 & 8,73 & 0,242 \\
\hline & Strongyloides fuelleborni & 3,48 & 5,93 & 0,159 & 6,12 & 4,05 & 3,57 & 4,64 & 0,74 & 6,06 & 3,66 & 0,176 \\
\hline & Strongyloides stercoralis & 4,75 & 6,3 & 0,411 & 3,4 & 6,76 & 7,14 & 4,64 & 0,464 & 6,06 & 5,07 & 0,606 \\
\hline & Trichuris trichiura & 12,03 & 10,37 & 0,528 & 12,24 & 9,46 & 7,86 & 15,23 & 0,18 & 13,85 & 9,58 & 0,11 \\
\hline & Trichostrongylus sp. & 1,27 & 2,97 & 0,146 & 3,4 & 0,68 & 2,14 & 1,99 & 0,428 & 3,03 & 1,41 & 0,177 \\
\hline & Dicrocoelium sp. & 5,06 & 3,7 & 0,426 & 6,8 & 5,41 & 2,86 & 2,65 & 0,248 & 6,06 & 3,38 & 0,124 \\
\hline & Enterobius vermicularis & 2,22 & 1,48 & 0,514 & 0,68 & 3,38 & 1,43 & 1,99 & 0,386 & 1,73 & 1,97 & 0,834 \\
\hline & Hymenolepis nana & 6,65 & 7,41 & 0,719 & 2,72 & 10,14 & 7,86 & 7,28 & 0,094 & 4,76 & 8,45 & 0,087 \\
\hline & Schistosoma mansoni & 1,58 & 6,3 & $0,003 *$ & 2,72 & 2,7 & 5 & 4,64 & 0,595 & 5,19 & 2,82 & 0,139 \\
\hline \multirow{10}{*}{$\begin{array}{l}\mathbb{0} \\
0 \\
0 \\
0 \\
0 \\
0 \\
0 \\
0 \\
0 \\
0\end{array}$} & Entamoeba coli & 48,73 & 51,11 & 0,566 & 48,3 & 49,32 & 50,71 & 50,99 & 0,926 & 51,95 & 48,45 & 0,408 \\
\hline & Entamoeba histolytica/dispar & 25,95 & 24,44 & 0,676 & 25,17 & 22,3 & 28,57 & 25,17 & 0,645 & 26,84 & 24,23 & 0,477 \\
\hline & Entamoeba hartmanni & 17,41 & 22,22 & 0,143 & 17,69 & 17,57 & 22,14 & 21,19 & 0,629 & 20,78 & 18,87 & 0,57 \\
\hline & Endolimax nana & 19,3 & 20 & 0,832 & 19,05 & 20,27 & 16,43 & 21,85 & 0,677 & 29,44 & 13,24 & $0,000 *$ \\
\hline & Iodamoeba butschlii & 20,89 & 18,52 & 0,473 & 24,49 & 21,62 & 18,57 & 14,57 & 0,189 & 22,08 & 18,31 & 0,263 \\
\hline & Balantidium coli & 5,38 & 9,63 & 0,049 & 10,2 & 7,43 & 4,29 & 7,28 & 0,296 & 10,82 & 5,07 & $0,009^{*}$ \\
\hline & Chilomastix mesnili & 17,72 & 18,15 & 0,893 & 23,81 & 17,57 & 17,86 & 12,58 & 0,102 & 18,61 & 7,32 & 0,723 \\
\hline & Giardia duodenalis & 7,91 & 10,37 & 0,301 & 7,48 & 6,08 & 12,14 & 10,6 & 0,233 & 12,99 & 6,48 & $0,007 *$ \\
\hline & Blastocystis sp. & 10,76 & 20,74 & $0,001 *$ & 20,41 & 13,51 & 15 & 12,58 & 0,25 & 13,42 & 16,62 & 0,294 \\
\hline & Total & 69 & 70 & & 68,71 & 66,67 & 74,29 & 68,46 & & 68,83 & 69,86 & \\
\hline
\end{tabular}

SP : Saison Pluvieus 
Tableau VI. Répartition des individus en fonction du statut parasitaire dans les différentes localité

\begin{tabular}{llll}
\hline & $\begin{array}{l}\text { Individus } \\
\text { non parasités } \\
\text { n (\%) }\end{array}$ & $\begin{array}{l}\text { Individus } \\
\text { monoparasités } \\
\mathbf{n}(\boldsymbol{\%})\end{array}$ & $\begin{array}{l}\text { Individus } \\
\text { polyparasités } \\
\mathbf{n}(\boldsymbol{\%})\end{array}$ \\
\hline Gouléako 1 & $2(2,85)$ & $5(7,14)$ & $63(93)$ \\
Gouléako 2 & $5(2,36)$ & $21(9,9)$ & $186(87,73)$ \\
Paulé-Oula & $9(3,75)$ & $18(7,5)$ & $213(88,75)$ \\
PNT & $2(3,12)$ & $3(4,69)$ & $59(92,19)$ \\
Total & $\mathbf{1 8 ( 3 , 0 7 )}$ & $\mathbf{4 7}(\mathbf{8 , 0 2})$ & $\mathbf{5 2 1}(\mathbf{8 8 , 9 )}$ \\
\hline
\end{tabular}

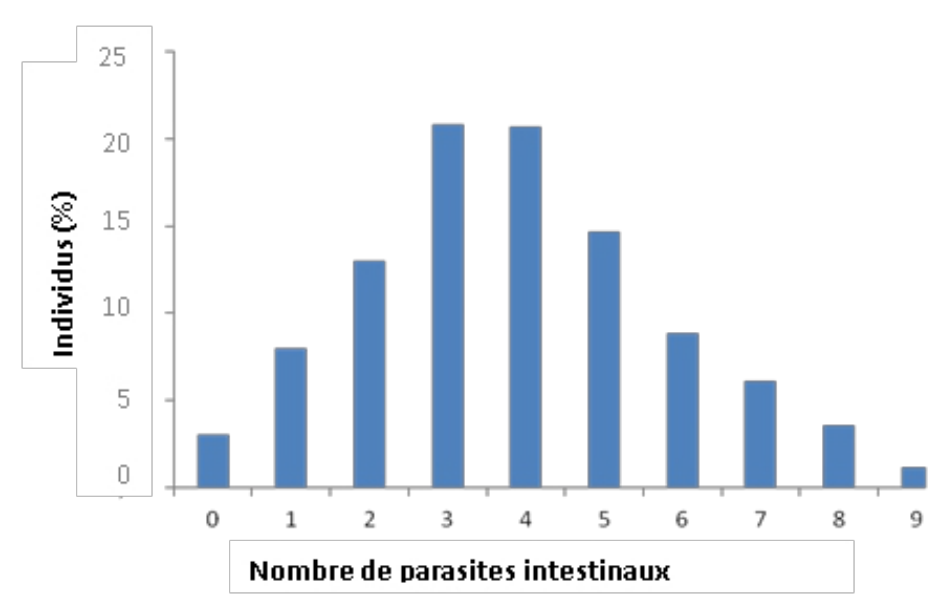

Figure 2. Nombre de parasites par individu dans l'ensemble des localités

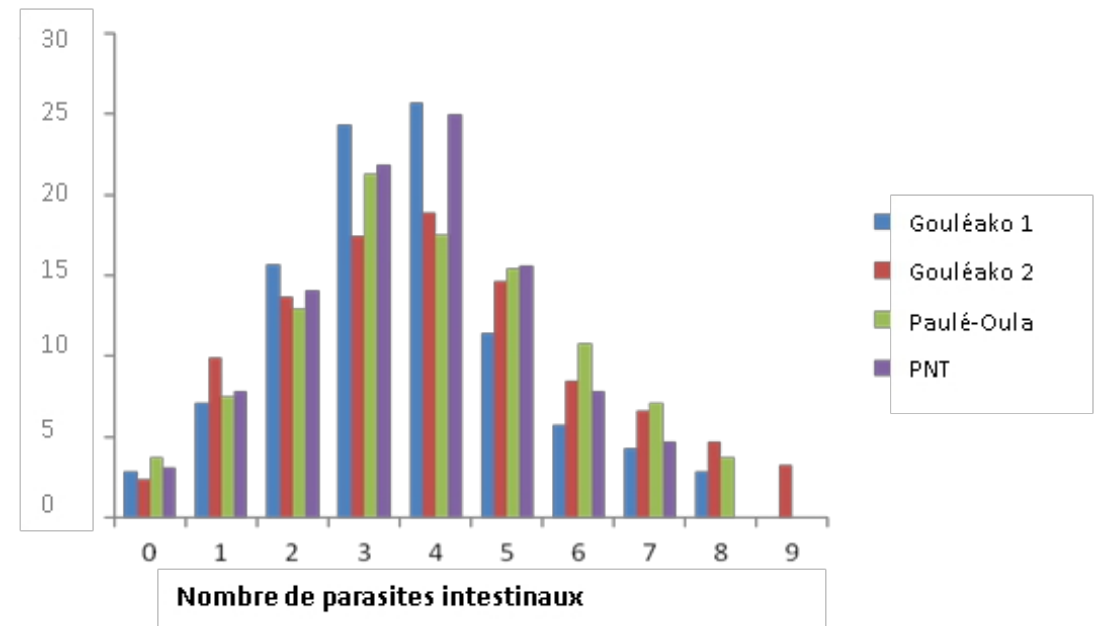

Figure 3. Nombre de parasites par individu dans chaque localité

\section{Discussion}

La faune parasitaire intestinale des populations de Gouléako 1, Gouléako 2 et Paulé-Oula d'une part, et, d'autre part, les chercheurs et les assistants de recherche travaillant à l'intérieur du parc national de Taï a été 
caractérisée au cours de cette étude. Les résultats indiquent des prévalences parasitaires élevées et comprises entre 64 et $80 \%$.

Une richesse spécifique et une diversité parasitaire importante ont été également enregistrées chez ces populations. 21 espèces de parasites intestinaux ont été identifiées. Ces résultats sont supérieurs à ceux obtenus dans les autres régions de la Côte d'Ivoire (Coulibaly et al., 2012; Yapi et al., 2014 ; Koné et al., 2015 ; Coulibaly et al., 2018). Bien que la majorité des espèces de parasites identifiés à Taï a déjà été observée lors de ces précédents travaux menés dans des populations d'autres régions de la Côte d'Ivoire, la faune parasitaire des populations humaines de Taï enregistre, en plus, Oesophagostomum sp., Capillaria Gen. sp. 2, Dicrocoelium sp., Trichostrongylus sp., Strongyloides fuelleborni, Balantidium coli.

En effet, ces villages de la région de Taï ne bénéficient pas de la connexion au réseau national de distribution d'eau potable. Chaque village possède, à l'exception du village de Gouléako 1, une seule pompe hydraulique pour tous les habitants. Cette situation favorise l'utilisation de différentes sources d'eau (eau de puits ou de marigots) pour la consommation et les besoins usuels et quotidiens. Ces problèmes d'eau seraient l'une des causes des prévalences parasitaires élevées dans cette région, comme c'est le cas pour le village de Gouléako 1 qui enregistre la prévalence globale d'infestations parasitaires la plus forte $(81,43 \%)$. De même, l'élevage porcin mis en place dans le passé dans des enclos mais présentement effectué « en plein air » serait l'une des causes de la prédominance de Balantidium coli à Paulé-Oula et Gouléako 2. Parmi les géohelminthes les plus répandus dans les populations de Taï figurent Ancylostoma sp. (24,74\%), Ascaris lumbricoides (11,95\%), Trichuris trichiura $(11,26 \%)$. Des travaux réalisés dans les régions du centre, de l'ouest et du sud-est de la Côte d'Ivoire, plus globalement en Afrique, sont arrivés aux mêmes conclusions (Raso et al., 2004; Tchuem Tchuenté, 2011; Coulibaly et al., 2012; Yapi et al., 2014 ; Coulibaly et al., 2018). Entamoeba coli, E. histolytica/dispar, E. hartmanni, Endolimax nana et Iodamoeba butschlii, sont les amibes prédominantes dans la population humaine de cette étude. La prévalence d'E. histolytica/dispar (25,26\%) est supérieure à celles enregistrées dans les départements d'Agboville et d'Adzopé (région sud-est), les départements de Man et de Duekoué (région ouest) (Eholié et al., 1999; Heckendorn et al., 2002; Ouattara et al., 2010). Quant aux amibes non pathogènes, de nombreux auteurs ont également démontré la prédominance de E. coli et E. nana (Penali et al., 1993; Adou-Bryn et al., 2001; Raso et al., 2005; Matthys et al., 2007). Bien qu'ils soient dépourvus de pouvoir pathogène, ces protozoaires constituent de très bons indicateurs du niveau d'hygiène et d'insalubrité (Monjour et al., 1998).

Une liaison significative a été observée entre le sexe et les prévalences d'Oesophagostomum sp., Schistosoma mansoni, Blastocystis sp. qui 
prédominent chez les hommes. La classe d'âge [4-7ans] est plus infestée avec une prévalence parasitaire cumulée de 74,29\%. Seule l'ascaridiose est significativement liée à l'âge chez les [3-6 ans]. Cette infestation diminue avec l’âge. La tendance à la géophagie plus développée chez les enfants, ainsi que l'acquisition progressive de l'immunité avec l'âge, expliqueraient ces résultats. En général, l'intensité moyenne des infestations dues aux géohelminthes change avec l'âge. Elle a une tendance convexe, augmentant dans l'enfance et diminuant à l'âge adulte. Les infestations à Ascaris lumbricoides et à Trichuris trichiura sont plus fréquentes et lourdes chez les enfants âgés de 5 à 15 ans. En revanche, l'intensité des infestations par les ankylostomes augmente de façon constante avec l'âge pour atteindre un pic chez l'adulte (Tchuem Tchuenté, 2011). A Taï, les prévalences des ankylostomes restent pratiquement constantes suivant l'âge.

Les résultats de cette étude confirment également que le polyparasitisme est commun aux communautés rurales de Taï comme c'est le cas dans d'autres régions à fortes infestations intestinales de Côte d'Ivoire et d'Afrique subsaharienne (Brooker et al., 2000; Raso et al., 2004; Ouattara et al., 2008; Coulibaly et al., 2012; Hürlimann et al., 2014).

Etroitement liés au péril fécal et à l'hygiène alimentaire, les parasites intestinaux dans leur grande majorité sont surtout fréquents chez les populations des pays tropicaux en voie de développement où l'hygiène fécale et corporelle est insuffisante.

Plusieurs travaux menés dans des populations rurales de Côte d'Ivoire ont démontré l'impact des bonnes pratiques d'hygiène (lavage des mains avant le repas et après les selles, utilisation de latrines, consommation d'eau potable) sur la réduction des infestations dues aux helminthes et aux protozoaires intestinaux (Raso et al., 2005; Raso et al., 2006; Ouattara et al., 2008 ; 2010; Schmidlin et al., 2013).

D’une façon générale, les populations pauvres des zones rurales sont exposées à de multiples risques d'infestations associées à la mortalité et la morbidité, à travers de multiples facteurs comme le manque d'eau potable, d'assainissement, les mauvaises conditions de logement. Selon Nematian et al. (2004), le faible niveau d'éducation et les mauvaises conditions socioéconomiques et hygiéniques des familles seraient de puissants déterminants des infestations. L'UNICEF, à travers son programme WASH (Eau, Assainissement et Hygiène), mène des interventions visant à améliorer la qualité de l'eau et son approvisionnement, tout en garantissant les conditions d'hygiène publique et en encourageant le lavage des mains avec du savon. Les méta-analyses de données de ce programme ont montré que les interventions réalisées étaient associées à une réduction du risque de transmission des helminthoses et des maladies infectieuses à l'homme avec un effet bénéfique 
sur la croissance des enfants (Ziegelbauer et al., 2012; Dangour et al., 2013; Strunz et al., 2014).

\section{Conclusion}

$\mathrm{Au}$ nombre des maladies tropicales négligées, les parasitoses digestives demeurent un problème majeur de santé publique dans les communautés des zones rurales pauvres. Le présent travail a permis de fournir la première base de données des parasites intestinaux chez les populations humaines riveraines du PNT.

Cette étude a permis d'identifier 21 espèces de parasites intestinaux, attestant une grande diversité spécifique chez ces populations. Les prévalences parasitaires globales sont élévées $(69,1 \%)$. Les acteurs du parc ont présenté la prévalence globale la plus faible, bien que la diversité parasitaire soit plus élevée dans cette population que dans les autres sites. Plusieurs parasites enregistrés suggèrent la présence potentielle de zoonoses. Les analyses moléculaires devront permettre d'évaluer la circulation de zoonoses parasitaires dans cette région.

\section{Remerciements}

Nous tenons à exprimer notre gratitude au Ministère de l'Enseignement Supérieur et de la Recherche Scientifique, au Ministère de l'Environnement et des Eaux et Forêts et à l'Office Ivoirien des Parcs et Réserves (OIPR) pour nous avoir accordé les autorisations pour la conduite de ce travail. Nous sommes reconnaissants au Centre Suisse de Recherches Scientifiques en Côte d'Ivoire et au Plateau Technique de Microbiologie des Hôpitaux Universitaires de Strasbourg (HUS) pour l'assistance technique. Nous remercions les Assistants de recherches du Taï Monkey Projet (TMP) pour leur aide dans la réalisation de nos travaux de terrain. Ce travail a été financé par le Programme d'Appui Stratégique à la Recherche Scientifique (PASRES, Project No. 48).

\section{References:}

1. Anoh, A. E., Akoua-Koffi, C., Couacy-Hymann, E., Pauly, M., Schubert, G., Mossoun, A., Weiss, S. Leendertz, S. A. J., Jarvis, M.A., Leendertz, F. H., \& Ehlers, B. (2015). Genetic identification of cytomegaloviruses in a rural population of Côte d'Ivoire. Virology Journal, 12, 155.

2. Anoh, A. E., Mossoun, A., Akoua-Koffi, C., Couacy-Hymann, E., Pauly, M., Leendertz, S. A., N'goran K. E., Schubert G., Weiss S., Hofmann J., Leendertz F. H., \& Ehlers, B. (2017). Seroprevalence of Cytomegalovirus Infection Among a Rural Population of Cote d'Ivoire. Viral Immunology 30, 54-57. 
3. Adou-Bryn, D., Kouassi, M., Brou, J., Ouhon, J., \& Assoumou, A. (2001). Prévalence globale des parasitoses à transmission orale chez les enfants à Toumodi (Côte d'Ivoire). Médecine d'Afrique Noire, 48, 394-398.

4. Blagg, W., Schloegel, E. L., Mansour, N. S., \& Khalaf, G. I. (1955). A new concentration technic for the demonstration of protozoa and helminth eggs in feces. American Journal of Tropical Medicine and Hygiene, 4, 23-28.

5. Brooker, S., Miguel, E. A., Moulin, S., Luoba, A. I., Bundy, D. A. P., \& Kremer, M. (2000). Epidemiology of single and multiple species of helminth infections among school children in Busia district, Kenya. East African Medical Journal, 77, 157-161.

6. Colwell, R. K. (2013). EstimateS: Statistical estimation of species richness and shared species from samples. Version 7.0, Persistent URL purl, Oclc. Org / estimates.

7. Coulibaly, G., Ouattara, M., Dongo, K., Hürlimann, E., Bassa, F. K., Koné, N., Essé, C., Yapi, R. B., Bonfoh, B., Utzinger, J., Raso, G., \& N'Goran, E. K. (2018). Epidemiology of intestinal parasite infections in three departments of south-central Côte d'Ivoire before the implementation of a cluster-randomised trial. Parasite Epidemiology and Control, 18, 63-76.

8. Coulibaly, T. J., Fürst, T., Silué, K. D., Knopp, S., Hauri, D., Ouattara, M., Utzinger, J., \& N'Goran K. E. (2012). Intestinal parasitic infections in schoolchildren in different settings of Côte d'Ivoire: effect of diagnostic approach and implications for control. Parasites and Vectors, 5, 135.

9. Dangour, A. D., Watson, L., Cumming, O., Boisson, S., \& Yan, C. (2013). Interventions to improve water quality and supply, sanitation and hygiene practices, and their effects on the nutritional status of children. Cochrane Database of Systematic Reviews, 8, CD009382.

10. Eholié, S. P., Coulibaly, M., Kra, O., Adou-Bryn, D., Yapi, Y. Z., Aoussi, E., Bissagnéné, E., \& Kadio, A. (1999). Diarrhée associée à une parasitose à Man (Côte d'Ivoire) : Aspects épidémiologiques, cliniques étiologiques et thérapeutiques. Médecine d'Afrique Noire, 46, 27-31.

11. Erdman, D. D. (1981). Clinical comparison of ethyl acetate and diethyl ether in the formalin-ether sedimentation technique. Journal of Clinical Microbiology, 14, 483-485.

12. Heckendorn, F., N'Goran, E. K., Felger, I., Vounatsou, P., Yapi, A., Oettli, A., Marti, H. P., Dobler, M., Traoré, M., Lohourignon, K., \& Lengeler, C. (2002). Species-specific field testing of Entamoeba spp. 
in an area of endemicity. Tansaction of the Royal Society of Tropical Medicine and Hygiene, 96, 521-528.

13. Hoppe-Dominik, B. (1995). L'état actuel des effectifs des grands mammifères dansl'ensemble du Parc National de Taï. GmbH-GTZ, DPN, Abidjan, Côte d'Ivoire, 66 p.

14. Hürlimann, E., Yapi, B. R., Houngbedji, A. C., Schmidlin, T., Kouadio, A. B., Silué, D.K., Ouattara, M., N'Goran, K. E., Utzinger, J., \& Raso, G. (2014). The epidemiology of polyparasitism and implications for morbidity in two rural communities of Côte d'Ivoire. Parasites and Vectors, 7, 81.

15. Kouassi, R. Y. W., McGraw, W. S., Yao, P. K., Abou-Bacar, A., Brunet, J., Pesson, B., Bonfoh, B., N'goran, E. K., \& Candolfi, E. (2015). Diversity and prevalence of gastrointestinal parasites in seven non-human primates of the Taï National Park, Côte d'Ivoire. Parasite, 22, 1-15.

16. Köndgen, S., Kühl, H., N'Goran, P. K., Walsh, P. D., Schenk, S., Ernst, N., Biek, R., Formenty, P., Mätz-Rensing, K., Schweiger, B., Junglen, S., Ellerbrok, H., Nitsche, A., Briese, T., Lipkin, W. I., Pauli, G., Boesch, C., \& Leendertz, F. H. (2008). Pandemic human viruses cause decline of endangered great apes. Current Biology, 18: 260-264.

17. Koné, S., Baikoro, N., N'Guessan, Y., Jaeger, F. N., Silué, K. D., Fürst, T., Hürlimann, E., Ouattara, M., Séka, M. C. Y., N’Guessan, N. A., Esso, E. L., Zouzou, F., Boti, L. I., Gonety, P. T., Adiossan, L. G., Dao, D., Tschannen, A. B., Stamm von, T., Bonfoh, B., Tanner, M., Utzinger, J., \& N'Goran, E. K. (2015). Health \& demographic surveillance system profile: the taabo health and demographic surveillance system, Côte d'Ivoire. International Journal of Epidemiology, 44, 87-97.

18. Leendertz, F., Ellerbock, H., Boesch, C., Couacy-Hymann, E., MätzRensing, K., Hakenback, R., Bergmann,C., Abaza, P., Junglen, S., Moebius, Y., Vigilant, L., Formenty, P., \& Pauli, G. (2004). Anthrax kills wild chimpanzees in a tropical rainforest. Nature, 430, 451-452.

19. Leonard, E., \& Ibo J. G. 1994. Appropriation et gestion de la rente forestière en Côte d'Ivoire. Politique Africaine, 53, 25-36.

20. Matthys, B., Tschannen, A. B., Tian-Bi, N. T., Comoe, H., Diabate, S., Traore, M., Vounatsou, P., Raso, G., Gosoniu, L., Tanner, M., Cisse, G., N'Goran, K. E., \& Utzinger, J. (2007). Risk factors for Schistosoma mansoni and hookworm in urban farming communities in western Côte d'Ivoire. Tropical Medicine and International Health, 12, 709723. 
21. Monjour, L., Bienvenu, N., Bonkoungou, B., \& Farhati, K. (1998). L'utilisation d'une eau potable associée ou non à des mesures de protection de l'environnement apporte-t-elle bénéfice à la santé et à l'état de santé des enfants en milieu périurbain tropical ? Ouagadougou Burkina Faso. Action pilote 6 du rapport final du Programme Alimentation en eau potable dans les quartiers périurbains et les petits centres, $34 \mathrm{p}$.

22. Mossoun, A, Pauly, M, Akoua-Koffi, C, Couacy-Hymann, E, Leendertz, S. A., Anoh, A. E., Gnoukpoho, H. A., Leendertz, F. H., \& Schubert, G. 2015. Contact to non-human primates and risk factors for zoonotic disease emergence in the Taï region, Côte d'Ivoire. EcoHealth, 12, 580-591.

23. Nematian, J., Nematian, E., Gholamrezanezhad, A., \& Asgari A. A. (2004). Prevalence of intestinal parasitic infections and their relation with socio-economic factors and hygienic habits in Tehran primary school students. Acta Tropical, 92, 179-186.

24. Ouattara, M., N'Guessan, N.A., Yapi., A., \& N'Goran, E. K. (2010). Prevalence and spatial distribution of Entamoeba histolytica/dispar and Giardia lamblia among schoolchildren in Agboville Area (Côte d'Ivoire). Eichinger D, ed. PLoS Neglected Tropical Diseases, 4(1), e574.

25. Ouattara, M., Silué, K. D., N’Guessan, A. N., Yapi, A., Barbara, M., Raso, G., Utzinger, J., \& N'Goran K. E. (2008). Prévalences et polyparasitisme des protozoaires intestinaux et répartition spatiale d'Entamoeba histolytica/Entamoeba dispar et Giardia intestinalis chez des élèves en zone rurale de la région de Man en Côte d'Ivoire. Cahiers d'études et de recherches francophones, 18, 215-222.

26. Penali, L. K., Broalet, E. Y., \& Koné, M. (1993). Helminthiases et protozooses intestinales de la femme enceinte en Côte d'Ivoire. Médecine d'Afrique Noire, 40, 354-356.

27. Pullan, R. L., Smith, J. L., Jasrasaria, R., \& Brooker, S.J. (2014). Global numbers of infection and disease burden of soil transmitted helminth infections in 2010. Parasites and Vectors, 7. 37.

28. Raso, G., Luginbühl, A., Acka-Douabéle, A. C., Tian-Bi, N. T., Silue, K. D., Matthys, B., Vounatsou, P., Wang, Y., Dumas, M. E., \& Holmes E. (2004). Multiple parasite infections and their relationship to selfreported morbidity in a community of rural Côte d'Ivoire. International Journal of Epidemiology, 33, 1092-1102.

29. Raso, G., Vounatsou, P., Gosoniu, L., Tanner, M., N’Goran, E. K., \& Utzinger J. (2006). Risk factors and spatial patterns of hookworm infection among schoolchildren in a rural area of western Côte d'Ivoire. International Journal for Parasitology, 36, 201-210. 
30. Raso, G., Utzinger, J., Silué, K. D., Ouattara, M., Yapi, A., Toty, A., Matthys, B., Vounatsou, P., Tanner, M., \& N'Goran E. K. (2005). Disparities in parasitic infections, perceived ill health and access to health care among poorer and les poor schoolchildren of rural Côte d'Ivoire. Tropical Medicine and International health, 10, 42-57.

31. Schmidlin, T., Hürlimann, E., Silue, K. D., Yapi, R. B., Houngbedji, C., Kouadio, A. B., Acka-Douabéle, A. C., Kouassi, D., Ouattara, M., Zouzou, F., Bonfoh, B., N'Goran, K. E., Utzinger, J., \& Raso, G. (2013). Effects of hygiene and defecation behavior on helminths and intestinal protozoa infections in Taabo, Cote d'Ivoire. PLoS One, 8, e65722.

32. Strunz, E. C., Addiss, D. G., Stocks, M. E., Ogden, S., \& Utzinger, J. (2014). Water, Sanitation, Hygiene, and Soil-Transmitted Helminth Infection: A Systematic Review and Meta-Analysis. PLoS Medecine, 11, e1001620.

33. Schweter, M. (1999). Interprétation des images SPOT. Evolution de la surface de forêt du Parc National de Taï, 1993-1998. Rapport PACPNT, San Pedro, Côte-d'Ivoire, 73 p.

34. Tchuem Tchuenté, L. A. (2011). Control of soil-transmitted helminths in sub Saharan Africa: Diagnosis, drug efficacy concerns and challenges. Acta Tropica, 120, 4-11.

35. Wilcox, B. A., \& Gubler, D. J. (2005). Disease ecology and the global emergence of zoonotic pathogens. Environmental Health and Preventive Medicine, 10, 263-272.

36. Yapi, R. B., Hürlimann, E., Schmidlin, T., Houngbedji, C., Ndri, B. P., Silue, K. D., Soro, G., Kouamé, N. F., Vounatsou, P., Fürst, T. N'Goran, K. E., Utzinger, J., \& Raso, G. (2014). Infection and coinfection with helminths and Plasmodium among school children in Côte d'Ivoire: Results from a National Cross-Sectional Survey. PLoS Neglected Tropical Diseases, 8, e291.

37. Young, K. H., Bullock, S. L., Melvin, D. M., \& Spruill, C. L. (1979). Ethyl acetate as a substitute for diethyl ether in the formalin-ether sedimentation technique. Journal of Clinical Microbiology, 10, 852853.

38. Ziegelbauer, K., Speich, B., Maüsezahl, D., Bos, R., \& Keiser, J. (2012). Effect of Sanitation on soil-transmitted helminth infection: Systematic review and Meta-analysis. PLoS Medecine, 9, e1001162. 